( $p<0,001)$, older age of onset $(p=0,019)$, higher levels of RF IgM $(p=0,027)$ and anti-CCP $(p<0,001)$. Development of persistent spontaneous remission negatively correlated with polyarthritis $(p=0,033)$, PF-positivity $(p=0,034)$, anti-CCP-positivity $(p=0,001)$. Positive seroconversion was observed: of RF in $10(4,7 \%)$ patients, 8 developed RA, of anti-CCP - in $3(1,4 \%)$ patients, all developed RA.

Conclusion: Seronegative oligoarticular disease and highly seropositive disease are different subtypes of UIA. Combination of seronegativity and oligoarticular disease $(n=52)$ associated with relatively rare development of RA (36,2\%) and high proportion of spontaneous remission $(22,4 \%)$. Patients who were highly positive ( $>3$ ULN) for both RF and anti-CCP developed RA in $97 \%$ of cases and never remitted spontaneously.

Disclosure of Interests: Elena Luchikhina Consultant of: Abbvie, Biocad, Sanofi, Celgene, Speakers bureau: Abbvie, Roche, Pfizer, Biocad, MSD, Sanofi, Johnson \& Johnson, Glaxo, UCB, Celgene, Novartis, Dmitry Karateev Consultant of: Abbvie, Pfizer, Biocad, Sanofi, Novartis, Lilly, Speakers bureau: Abbvie, Roche, Pfizer, Biocad, MSD, Sanofi, Johnson \& Johnson, Glaxo, UCB, Celgene, Novartis, Lilly, Bayer, Alexander Novikov: None declared, Galina Lukina Speakers bureau: Novartis, Pfizer, UCB, Abbvie, Biocad, MSD, Roche, Elena Aleksandrova: None declared, Natalia Demidova: None declared

DOI: 10.1136/annrheumdis-2020-eular.6043

\section{FRI0542 OBTAINING HIGH POSITIVE PREDICTIVE VALUES FOR THE DEVELOPMENT OF CLINICALLY APPARENT ARTHRITIS IN PATIENTS PRESENTING WITH CLINICALLY SUSPECT ARTHRALGIA; IS IT FEASIBLE?}

X. Matthijssen ${ }^{1}$, B. Van Dijk ${ }^{1}$, F. Wouters ${ }^{1}$, E. Niemantsverdriet ${ }^{1}$, A. Van der Helm-van Mil ${ }^{1,2}{ }^{1}$ Leiden University Medical Center (LUMC), Leiden, Netherlands; ' ${ }^{2}$ rasmus MC, Rotterdam, Netherlands

Background: The hypothesis that initiation of DMARD-treatment before arthritis becomes apparent could permanently modulate the disease process, such that persistent RA is prevented, is being studied in several ongoing trials. Essential for such studies is the ability to accurately predict clinically apparent inflammatory arthritis (IA). However there are two hurdles: first, it is insufficiently known whether it is possible to obtain high positive predictive values (PPV) in patients presenting with clinically suspect arthralgia (CSA). Second, none of current predictive models is validated in independent cohorts. We here aimed to evaluate the first question, incorporating improved markers of MRI-detected subclinical inflammation that were recently identified but have not yet been combined with other known predictors.[1]

Objectives: To assess the feasibility of achieving high PPVs in prediction of IA-development in patients with CSA by combining clinical, laboratory and imaging parameters.

Methods: 580 patients with CSA were consecutively included in the Clinically Suspect Arthralgia (CSA)-cohort and followed on the development of IA, determined by physical examination of joints. Unilateral contrast-enhanced 1.5 Tesla MRIs were made of MCP(2-5), wrist and MTP(1-5)-joints at baseline and scored in line with the RAMRIS. The number of locations with subclinical inflammation $(0 / 1-2 / \geq 3)$ and the presence of MCP peritendinitis were defined as described previously.[1] Other studied clinical and laboratory variables were based on the literature: initial localisation of complaints (small/ large joints), functional disability (health assessment questionnaire (HAQ) 21), ACPA-positivity (Anti-CCP2), RF-positivity (IgM-RF) and elevated CRP. $[2,3]$ LASSO Cox regression with a 10-fold cross-validated shrinkage parameter was used for predictor selection. Regression coefficients were rounded to the nearest number ending in .5 or .0 and multiplied by two, resulting in a weighted score. Kaplan Meijer curves were used to obtain PPVs of this weighted score and the area under the curve (AUC) was determined at 2-year follow-up.

Results: Mean age was $44,78 \%$ was female, and $18 \%$ progressed to IA within 2 years. The following parameters were selected with LASSO: RF-positivity, ACPA-positivity, $\mathrm{HAQ} \geq 1,>2$ locations of subclinical inflammation and presence of MCP-extensor peritendinitis. Based on the beta of LASSO-regression, patients were assigned 2 points for the risk-factors ACPA-positivity and $>2$ locations of subclinical inflammation, 1 point for RF-positivity and presence of MCP-extensor peritendinitis and 0 points for $\mathrm{HAQ} \geq 1$. Kaplan Meijer curves show PPVs of $8 \%$, $9 \%, 30 \%, 54 \%, 73 \%, 79 \%$ and $86 \%$ at two years (Figure 1 ). This model yielded an AUC of 0.79 .

Conclusion: High PPVs for IA-development can be achieved in patients with CSA by weighting a combination of known predictors. Although encouraging,

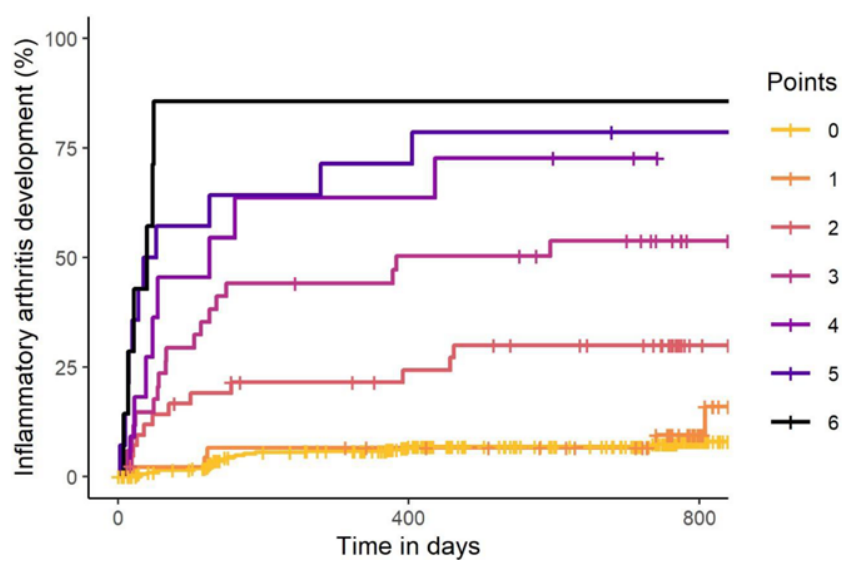

Figure 1. Kaplan Meijer curves on inflammatory arthritis development stratified for number of points based on LASSO regression. Legend: Points were based on the regression coefficients yielded by Cox LASSO-regression. 2 points were assigned for the risk factors ACPA-positivity and $>2$ locations of subclinical inflammation and 1 point was assigned for RF-positivity and presence of MCP-extensor peritendinitis.

these data are based on one observational cohort study and have not been validated in independent cohorts, limiting the relevance. To support future research in the field of arthralgia, it is needed that different research groups work together to come to risk estimations that are validated and accepted.

References:

[1] Matthijssen XME et al. ART 2019;21(1):249-.

[2] van Steenbergen HW et al. ART 2014;16(2):R92.

[3] ten Brinck RM et al. RMD Open 2017;3(1):e000419.

Disclosure of Interests: None declared

DOI: 10.1136/annrheumdis-2020-eular.1697

\section{FRI0543 BIRTH REGISTRY OF WOMEN WITH SYSTEMATIC LUPUS ERYTHEMATOSUSAND COURSE OFTHE DISEASE DURING FIRST YEARS POST-PARTUM-THE GREEK EXPERIENCE}

S. Ntali ${ }^{1}$, L. Pantazi ${ }^{2}$, K. Boki ${ }^{2}$, D. Nikolopoulos ${ }^{3}$, A. Fanouriakis ${ }^{3}$

D. Dimopoulou ${ }^{4}$, I. Kallitsakis ${ }^{5}$, C. Papagoras ${ }^{6}$, V. Dania ${ }^{2}$, E. Emmanouilidou ${ }^{7}$,

G. Bertsias ${ }^{7} .{ }^{1}$ private practitioner, Thessaloniki, Greece; ${ }^{2}$ Sismanoglio GUH,

Rheumatology Unit, Athens, Greece; ${ }^{3} \mathrm{GUH}$ Attico, 4th Internal Medicine

University Clinic, Athens, Greece; ${ }^{4}$ Aristoteles University, 4th Internal Medicine

University Clinic, Thessaloniki, Greece; ${ }^{5}$ Private Practitioner, Chania, Greece:

${ }^{6}$ Democritus University of aLEXANDROUPOLI, 1st Department of Internal medicine, Alexandroupoli, Greece; ${ }^{7}$ University Of Crete, Rheumatology and Immunology Department, Herakleion, Greece

Background: Pregnancy in women with SLE Systematic Lupus Erythematosus (SLE) has been related with adverse events both in the mother and the foetus. 1 Many studies have reported relapse of the disease during the pregnancy and post-labour, while others have not confirmed this finding.2To this end, most of these results originate from retrospective studies with patients of diverge ethnicities.

Objectives: To record the Greek experience with pregnancies in mothers with SLE and their outcomes, as well as the course of the disease during first year post labor.

Methods: This is a prospective, multicentre, observation study lasting three years. Women diagnosed with SLE who became pregnant consented to be monitored by their treating Rheumatologist. A structured questionnaire is used for monitoring at the beginning of pregnancy (positive pregnancy test) and at least every 3 months thereafter, depending on the course of the disease and pregnancy, until one year after childbirth.

Results: A total 64 women and 81 pregnancies were recorded (1.27 pregnancies per patient). Patient's age at conception was $32.8 \pm 5.9$ years (mean \pm standard deviation). Thirteen patients $(20.3 \%)$ had past history of nephritis. Regarding pregnancy outcomes, $62(76.5 \%)$ pregnancies ended in live births, miscarriages during $1^{\text {st }}, 2^{\text {nd }}$ and $3^{\text {rd }}$ trimester occurred in $13(16 \%)$. Six pregnancies were lost to followup. Prematurity occurred in 28 live births (45.1\% in total), $26-32 w(3.2 \%)$, $32-36 w(22.5 \%),<37 w(19.3 \%)$. No cases of preeclampsia occurred. Mean age of birth 36.9 weeks and mean birth weight $2750 \mathrm{gr}$. The majority $(72.5 \%)$ of deliveries were performed by caesarean section. In terms of disease activity,

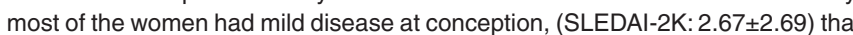


declined during $1^{\text {st }} / 2^{\text {nd }}$ pregnancy trimester (SLEDAI-2K:1.91 $\left.\pm 2.09,1.70 \pm 2.22\right)$ ) but increased during the $1^{\text {st }}$ and $2^{\text {nd }}$ trimester post labor (SLEDAI-2K: $2.47 \pm 4.29$ and $2.52 \pm 3.2$ ).

Conclusion: This is the first Greek inception cohort with prospective monitoring of pregnant SLE patients. Adverse outcomes occur with prematurity being the most frequent. In our cohort disease activity tends to increase during $1^{\text {st }}$ and $2^{\text {nd }}$ trimester post-labor without serious relapses. Vigilant monitoring during pregnancy and post-labour is advised.

References:

[1] Bundhun PK, Soogund MZ, Huang F. Impact of systemic lupus erythematosus on maternal and fetal outcomes following pregnancy: A meta-analysis of studies published between years 2001-2016. J Autoimmun 2017;79:17-27. [https://doi. org/10.1016/j.jaut.2017.02.009] [PMID: 28256367]

[2] Wei S, Lai K, Yang Z, Zeng K. Systemic lupus erythematosus and risk of preterm birth: a systematic review and meta-analysis of observational studies. Lupus 2017;26:563-71. [https://doi. org/10.1177/0961203316686704] [PMID: 28121241]

Acknowledgments: Hellenic Rheumatology Association

Disclosure of Interests: Stella Ntali: None declared, Lina Pantazi: None declared, Kyriaki Boki: None declared, Dionysis Nikolopoulos: None declared, Antonis Fanouriakis: None declared, Despoina Dimopoulou: None declared, loannis Kallitsakis Grant/research support from: MSD, Speakers bureau: Genesis pharma, Bristol-Myers Squibb, CHARALAMPOS PAPAGORAS: None declared, Vasiliki Dania: None declared, Evgenia Emmanouilidou: None declared, George Bertsias Grant/research support from: GSK, Consultant of: Novartis

DOI: 10.1136/annrheumdis-2020-eular.6195

\section{FRI0544 THE EFFECT OF PATERNAL EXPOSURE TO IMMUNOSUPPRESSIVE DRUGS ON SEXUAL FUNCTION, REPRODUCTIVE HORMONES, FERTILITY, PREGNANCY AND OFFSPRING OUTCOMES: A SYSTEMATIC REVIEW}

L. F. Perez ${ }^{1}$, R. Dolhain ${ }^{1}$, S. Vorstenbosch ${ }^{2}$, W. Bramer ${ }^{3}$, E. Van Puijenbroek ${ }^{2,4}$, J. Hazes ${ }^{1}$, B. Te Winkel' ${ }^{2}{ }^{1}$ Erasmus MC, University Medical Center, Rheumatology, Rotterdam, Netherlands; ${ }^{2}$ Netherlands Pharmacovigilance Centre Lareb, 's-Hertogenbosch, Netherlands; ${ }^{3}$ Erasmus MC. University Medical Center, Medical Library, Rotterdam, Netherlands; ${ }^{4}$ University of Groningen, Groningen Research Institute of Pharmacy, PharmacoTherapy, Epidemiology and Economics, Groningen, Netherlands

Background: Information regarding the possible influence of immunosuppressive drugs on male sexual function and reproductive outcomes is scarce. Men diagnosed with immune-mediated diseases and a wish to become a father represent an important neglected population since they lack vital information to make balanced decisions about their treatment.

Objectives: To systematically review the literature for the influence of paternal immunosuppressive drug use on many aspects of male sexual health, such as sexual function, fertility, pregnancy outcomes and on their offspring health outcome.

Methods: A systematic literature search was performed in the bibliographic databases: Embase (via Elsevier embase.com), MEDLINE ALL via Ovid, Cochrane Central Register of Trials (via Wiley) and Web of Science Core Collection. Additionally, Google Scholar and the Clinical trial registries of Europe and the USA were searched. The databases were searched from inception until August 31th 2019. The searches combined keywords regarding male sexual function and fertility, pregnancy outcomes and offspring's health with a list of immunosuppressive drugs. Studies were included if they were published in English and if they included original data on male human exposure to immunosuppressive drugs.

Results: A total of 5867 references were identified among which we identified 163 articles fulfilling the eligibility criteria. Forty nine articles included pregnancy and offspring outcomes and 116 articles included sexual health outcomes. With the exception of large Scandinavian cohorts, most of the identified articles included a small number of participants. While a clear negative effect on sperm quality was evident for sulfasalazine and cyclophosphamide a dubious effect was identified for colchicine, methotrexate and sirolimus. In 3 articles exposure to TNF-a inhibitors in patients diagnosed with ankylosing spondylitis resulted in improved sperm quality. The information regarding pregnancy and offspring outcomes was scant but no large negative effect associated with paternal immunosuppressive drug exposure was reported.

Conclusion: Evidence regarding the safety of immunosuppressive drugs in men with a wish to become a father is inconclusive. The lack of standardization on how to evaluate and report male sexual function, fertility and reproduction as study outcomes in men exposed to immunosuppressive drugs is an important contributor to this result. Future research on this topic is needed and should be preferably done using standardized methods.

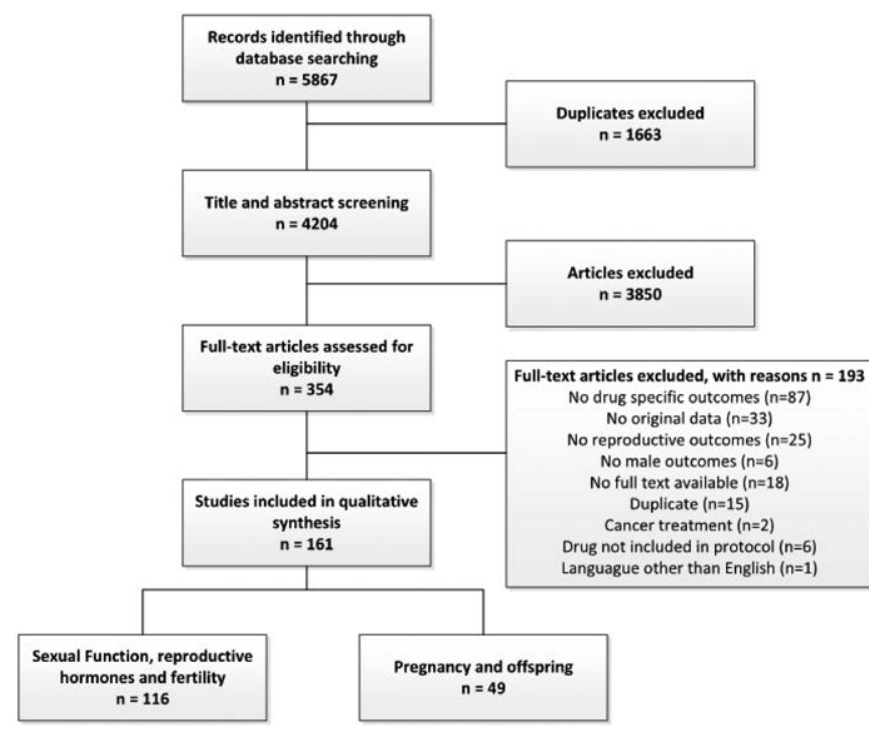

Figure 1. Flow diagram for study selection.

Disclosure of Interests: Luis Fernando Perez: None declared, Radboud Dolhain Grant/research support from: unrestricted grant from UCB Pharma, Saskia Vorstenbosch: None declared, Wichor Bramer: None declared, Eugène van Puijenbroek: None declared, Johanna Hazes: None declared, Bernke te Winkel: None declared

DOI: 10.1136/annrheumdis-2020-eular.3092

\section{FRI0545 1 A META-ANALYSIS OF GIANT CELL ARTERITIS TEMPORALLY AND ACROSS REGIONS}

D. Semenov ${ }^{1}, \mathrm{~K}_{\text {. LI }}{ }^{1}, \mathrm{M}$. Turk ${ }^{1}$, J. Pope ${ }^{1}$ on behalf of Schulich School of Medicine \& Dentistry. ${ }^{1}$ Schulich School of Medicine \& Dentistry, London, Canada

Background: Giant cell arteritis (GCA) is an immune-mediated disease of the large vessels, and occurs in adults over 50 years old ${ }^{1}$. It is the most commonly seen form of chronic vasculitis and is associated with significant rates of morbidity $^{2}$. This meta-analysis examines the geographical and temporal epidemiology of GCA, including incidence, prevalence and mortality.

\section{Objectives:}

1.To identify changes in incidence rate, prevalence, and mortality rate over time 2.To compare these rates between geographic regions around the world Methods: A systematic review of the English literature was conducted using the EMBase, Scopus and PubMed databases. Articles were included if they were cohort or cross-sectional studies with 50 or more patients with GCA and reported on population, location and time-frame parameters. Articles on mortality were included if they compared mortality to age and gender matched population. Review articles, case-control studies and case series were excluded. Two reviewers extracted data and a third verified inclusion of studies. Study quality was assessed by using the Strengthening the Reporting of Observational Studies in Epidemiology (STROBE) checklist. Mortality rate was standardized across cohorts to deaths per 1000 people per year.

Results: Of the 3569 citations identified by the literature search, 107 were included in analysis. The pooled incidence of GCA internationally was $10.00[9.22,10.78$ cases per 100000 people over 50 years old (Figure). This incidence was highest in Scandinavia 21.57 [18.90, 24.23], followed by North and South America 10.89 [8.78, 13.00], Europe 7.26 [6.05, 8.47], and Oceania 7.85 [1.48,17.19]. Nine studies reported prevalence. Pooled prevalence from these 9 was $51.74[42.04,61.43]$ cases per 100000 people over 50 years old. Overall, pooled mortality was 20.44 $[17.84,23.03]$ deaths/1000 per year. Mortality had a generally decreasing trend over the years of publication.

Conclusion: The incidence of GCA varies regionally almost 3-fold. Likely genetic and environmental factors may explain this trend. Incidence and prevalence are important for tracking the efficacy and side effects of current therapies, as well as planning for the costs of biologic treatment. 